\title{
Human Tracing using Location based System
}

\author{
G.S. Mate ${ }^{1}$, Sumit Gahire ${ }^{2}$, Rameshwar Mane ${ }^{3}$, Abhay Sharma ${ }^{4}$, Ravikiran Waghmare ${ }^{5}$ \\ Professor, Dept of IT, Rajarshi Shahu College of Engineering, Savitribai Phule Pune University, Pune, India ${ }^{1}$ \\ Student, Dept of IT, Rajarshi Shahu College of Engineering, Savitribai Phule Pune University, Pune, India ${ }^{2,3,4,5}$
}

\begin{abstract}
Now-a-days crimes are getting worse day by day especially when it's comes to women and children. Nobody feels safe while travelling alone and mainly to beat abandoned place. However, we recognize that there is need to provide necessary system/devices to overcome these issues. Various applications and algorithm are proposed to overcome these issue by providing the android app to user like Abhaya, Raksha, and Fight back through which user can tap the button on app whenever they feel vulnerable at public place or when the need arises. These system uses GPS for tracing the location and these app can send the real time and continuous location of the user. Several algorithm are developed to enhance the location accuracy. All these devices has result in the reduction of crime and improve the human safety. The objective of this survey paper is to propose a system through which a vulnerable user can get help instantly.
\end{abstract}

Keywords: GPS, GSM, Location, Latitude, Longitude.

\section{INTRODUCTION}

The crimes is increasing day-by-day all over the world and when it comes to a developing country like India it is major issue because of the unavailability of advance devices and technology, most of the time the help seeker does not get the services ASAP and that result in serious crime. According to the World Health Organization around 35\% Women across the world are the victim of sexual violence. In India women is assaulted in every three minutes. A Woman is weaker when compared to men physically, in an emergency situation and requires a helping person to relieve them. The best way to reduce the violent crime (Sexual assault, rape, domestic violence, robbery) is to identify and call on resources to help them out of dangerous situations. Many system are available in the market which uses GPS devices to locate an object. The Global Positioning System (GPS) allows properly equipped users to determine their position based on the measured pseudo-ranges to at least four satellites, GPS positioning accuracy is limited by measurement errors. The GPS greatly increases the accuracy and reduces the cost and complexity of navigation for land, marine, and air and space users. Under normal operating conditions, it is able to provide positioning accuracies in the range of 15-

$25 \mathrm{~m}$. But the accuracy of positioning can be increased using Differential Global Positioning System (DGPS) technique.

Many applications are developed that works mainly on the android and iOS operating system which uses GSP to locate the location and internet to send the location traced by the GPS to the preprogrammed contacts to get the necessary help whenever required. The problem with these system are that the user has to open an application And tap the button which is time consuming and also the system requires internet to send location information. To overcome these Challenges various system are proposed.

\section{EXISTINGSYSTEM}

A. Women and Children's Security Based Location Tracking System [1].

A new system for location based tracking system to overcome the tracking system using Wi-Fi as it required Wi-Fi device all the time for the communication .The system is totally controlled by needy person. The architecture of this system consists of sim card for making communication through radio channels, GPS transceiver for getting $2 \mathrm{D}$ or $3 \mathrm{D}$ location information from satellite .It required three satellite to get the $2 \mathrm{D}$ location and four satellite to get the 3D location. GPS uses a programmed UART (Universal Asynchronous Receiver/Transmitter) microcontroller to pass the location information into the link parameters and into the emergency button. The UART is use for processing the system .The GSM gives a gigantic baud rate with UART thus resulting in a high performance. With GPS it gets the location and send the data by using GPS module. The data format of the location is in the form of link (http://maps.google.com/? $\mathrm{t}=\mathrm{k} \& \mathrm{q}=$ LATITUDE, LONGITUDE) which is send to contacts. The receiver needs to install the Google map in order to get the location of the helper carrying thedevice.

B. Performance of Differential GPS Based on a Real-Time Algorithm Using SMS Services of GSM Network[2].

The author uses Differential Global Positioning System (DGPS) technique to improve the accuracy of position. The DGPS in this system is divided into two parts - the Reference Station (RS) and Mobile Station (MS).The RS Contains a GPS receiver and a computer with a software to perform the execution and then it sends the data to the MS using the GSM module. The MS could be anywhere within a circle of radius of $50 \mathrm{~km}$ around the RS. The MScontains two receiver, the first receiver is GPS receiver which takes and represents the position co-ordinate of MS and the second receiver is received by GSM modem as 
SMS andit shows the Differential data (DD). With the help of GSM the differential data which has been processed and calculated by the RS is send from RS to MS .The system was tested and a horizontal accuracy of $0.44 \mathrm{~m}$ and a vertical accuracy of $0.55 \mathrm{~m}$ were obtained. The system can operate with $\mathrm{n}$ numbers of mobile station and each MS can request the DD from the RS at any time via SIM service. The system can be implemented on a smart-phone instead of PC to make it handier infuture.

C. Geometry Motion Based Positioning Algorithm for Mobile Tracking in NLOS Environments [3].

The author aim to improve the positional accuracy of network based positioning system in severe non-line-ofsight (NLOS) and multipath environments. The implementation is done by using a two-step position determination method and a one-step method. Tominimize the cost function we perform an optimization method that takes the NLOS error into consideration so as to reduce the NLOS effect. Heading angle and velocity details is employed to adjust the primary position estimates to advance the position precisely.

The two-step position determination method is divided into two step which includes optimization with NLOS mitigation and geometry and motion based adjustment. After this process one-step position determination method is executed in which it propose a position determination scheme which jointly handles the two processes in one step. Later conductingthe two method it was observed that it is best suited in the circumstances where severe NLOS and multipath propagations exist. It was proved through simulation that the projected algorithm can improve the positioning accuracy compared to the presentalgorithms.

\section{GPS-GSM Based Tracking System [4].}

A tracking system which contains single-board embedded system that is equipped with GPS and GSM modemsalong with ARM processor that is installed in the vehicle.During object motion, its location can be sent by SMS. Tracking system inform where vehicle is and where it has been, how longer it has been there. It acquired, monitored and transmitted the position latitude, longitude, time to management center either at fixed interval or on demand. Microcontroller acquired and processed the positioneddata from GPS module. The GPS receiver of vehicle terminal received and resolved the navigation message Broadcasted by GPS position satellites, computed longitude andlatitude of vehicle coordinates, transformed into GSM message From by GSM communication controller, and sent message to monitoring center via the GSM network.

\section{E. A Mobile Application for Women's Safety:[5].}

Author concluded various mobile apps for women security like iMac, VithU, Nirbhaya which sends message to preselected contacts after pressing power button twice or some apps sends message after shaking a phone. The message contains user's GPS location. But proposed app Wos App is directly tie-up with local police and it is open source. It is developed using Rapid Application Development (RAD) model. By using this app user can store her name and number as well as name and number of her emergency contacts. In emergency case user can press the PANICicon on the app or can shake her phone to activate the trigger. After pressing PANIC icon or shaking phone it send the message to police which contains user's location and her emergency contacts. Then police will inform to her emergency contacts and by using Google map finds exact location of user. Immediately after the message is sent application automatically places a call to a dedicated helpline provided during the initial phase of the application by the Mangalore police. After getting user's location police is dispatch form nearest location of user's. But user must have to shake a phone 40 times within 8second.

F. Design and Implementation of Safety Armband for Women and Children using ARM7[6].

A safety armband to get help in case of emergency for children and women. The existing system detects the emergency by the behavior of the person which is categorized into two types happy and sad. The author proposed system to overcome this problem of false behavior detection and starting emergency help. In this an automatic device is implemented which will start sending the panic message and live video for the help to the police control room and a mobile preregistered mobile number. It has three options to start to device to get help by using a button, using flex sensor or fall detector sensor.

If the button is pressed then the device will start streaming the video and send the location details to the control room when the button is pressed or the sensors meet the criteria required for the emergency case. The messages will be sent after a specific time interval of $30 \mathrm{sec}$ with the updated details. The accuracy of the GPS is up to 40 meter. GPS module is used to get the exact location and GSM modules end the location in form of longitude and latitude to the desired mobile numbers.

G. ABHAYA: an Android app for safety women[7].

A system application for android users to track using various applications. It uses GPS to get the exact location of the person in trouble. Existing android applications does not have the real time location tracking system. This problem of the existing system is overcome in this proposed system. This module sends the message containing the URL of the location of the victim to the registered contacts.

When victim click on the app it checks if the required system Settings are enabled if not then it will enable all the system settings. It gets the location of person with help of GPS module and send the message containing Coordinates in the form of URL to the registered contact numbers for help. The received URL spots the exact location of the person. The location is sent to registered contacts after every 5 minutes. To stop sending this SMS we have to stop the application. 
Vol. 6, Issue 1, January 2017

H. Real Time Vehicle Tracking System Based on ARM7 GPS and GSM Technology [8].

Various existing vehicle tracking system which are available but they are application specific, region specific and they sent a message which contains the location of vehicle. Location is in the form of longitude and latitude so they require third party help for getting understandable and cleared exact location. The proposed system consist of hardware and GUI application for displaying actual location of vehicles. System is divided into two parts one is transmitter and other is receiver. Transmitter contains GPS receiver, GSM module and microcontroller. GPS receives location data from satellite and convert this data into data string of NMEA 0183 format. This format contains longitude, latitude, altitude, speed and other satellite information. ARM7 microcontroller is used whichreceives NMEA string from GPS and collect only latitude and longitude. Now this latitude and longitude readings are sent to receiver with the help of GSM module. At the receiver side, receiver receives the message which contains the longitude and latitude in the form of NMEA 0183 format. Receiver entered this readings into GUI application made in MATLAB. This GUI application convert this readings from NMEA 0183 format to degree format which is suitable for Google earth. After that Google earth is called by an algorithm which placed these reading on it. Then finally Google earth display the location of vehicle on map.

\section{CONCLUSION}

This paper reviews different approaches for women and children safety through various devices and application proposed. Different algorithm is used to improve the accuracy of location. All the system uses the location based system to locate the object. The Google maps provide the best accuracy and locationtracking.

Development of such devices has reduce the crime in large Numbers but these devices is time consuming and requires internet. To overcome that we are developing a devices that does not requires internet at user end and takeslesser Time as user only need to tap the button.

\section{REFERENCES}

[1] Dr.Velayutham. R, Sabari.M, ErkkiSutinen, SornaRajeswari.M, “An Innovative Approach for Women and Children's Security Based Location Tracking System "2016 International Conference on Circuit, Power and Computing Technologies [ICCPCT] , 978-15090-1277-0/16/\$31.00 @2016IEEE

[2] Dr. Asaad Al-Hindawi, Majeed Nader, "Performance of Differential GPS Based on a Real-Time Algorithm Using SMS Services of GSM Network" 2012 International Conference on Future Communication Networks, 978-1-4673-0260-9/12 \$31.00 @ 2012 IEEE

[3] Kegen Yu and ErykDutkiewicz, "Geometry and Motion Based Positioning Algorithms for Mobile Tracking in NLOS Environments", reviewed at the direction of IEEE Communications Society subject matter experts for publication in the IEEE Globecom 2010 proceedings, 978-1-4244-5638-3/10/\$26.00@ 2010 IEEE

[4] Abid khan, Ravi Mishra, "GPS - GSM Based Tracking System”, International Journal of Engineering Trends and Technology-
Volume3Issue2- 2012, ISSN: 2231-5381

[5] Dhruv Chand, Sunil Nayak, Karthik S. Bhat, Shivani Parikh, Yuvraj Singh, AmitaAjith Kamath National Institute of Technology Karnataka, Surathkal Karnataka, "A Mobile Application for Women's Safety: WoSApp", 978-1-4799-8641-5/15/\$31.00

[6] Glenson Toney, Dr. FathimaJabeen, Puneeth S, "Design and Implementation of Safety Armband for Women and Children using ARM7”, 978-1-4799-8371-1/15/\$31.00 c 2015 IEEE

[7] Pradip V Mistary, R H Chile, "Real Time Vehicle Tracking System Based on ARM7 GPS and GSM Technology", IEEE INDICON 2015 1570187057, 978-1-4673-6540-6/15/\$31.00 @2015 IEEE

[8] Ravi SekharYarrabothu, Bramarambika Thota, "ABHAYA: AN ANDROID APP FOR THE SAFETY OF WOMEN", IEEE INDICON 2015 1570191849, 978-1-4673-6540-6/15/\$31.00 C2015 IEEE.

\section{BIOGRAPHY}

Gitanjali S. Mate is a Assistant Professor in Information Technology Department of Rajarshi Shahu College of Engineering Tathawade, Pune. She received Master of Engineering degree in Pune institute of Computer Technology from Savitribai Phule University Pune and her research interests are Cloud Computing. 